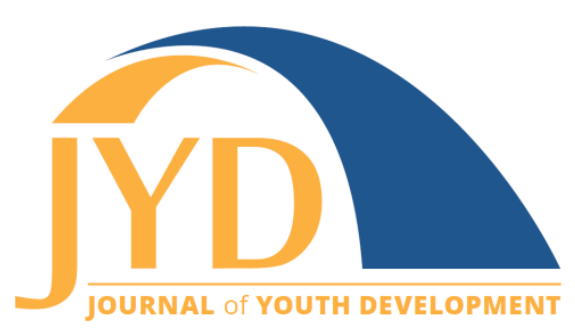

http://jyd.pitt.edu/ | Vol. 15 Issue 3 DOI 10.5195/jyd.2020.949 | ISSN 2325-4017 (online)

\title{
Optimizing Adolescent Health Behavior Programs
}

\section{Nicole F. Kahn}

National Academies of Sciences, Engineering, and Medicine

nkahn@nas.edu

\begin{abstract}
This article summarizes the key messages and recommendations from the recent National Academies report, Promoting Positive Adolescent Health Behaviors and Outcomes: Thriving in the $21^{\text {st }}$ Century. The first section outlines important definitions and frameworks that guided the committee's process. Next, the article describes the results of the committee's systematic review, which aimed to identify the core components of programs and interventions that are effective across a variety of adolescent health behaviors and outcomes. The final section of this article presents a subset of the committee's recommendations and promising approaches that can be useful to the diverse readership of this journal.
\end{abstract}

Key words: adolescent health, risk behavior, optimal health, core components, programs, interventions

\section{Introduction}

Adolescence is a critical developmental period when youth develop the knowledge, attitudes, and skills that will help them to thrive throughout life. Adolescents may learn these skills through prevention or intervention programs, which capitalize on the formative changes that occur during adolescence. However, no program is "one-size-fits-all," and too often these programs target specific risk behaviors instead of considering the needs of the whole person. These types of programs fail to understand the diverse experiences of youth as they move from adolescence through adulthood, and rarely take into account the context, communities, resources, or individual differences (e.g., age, race/ethnicity, socioeconomic status, gender identity, disability, etc.) that affect these experiences.

(cc) $\mathrm{EY}$ New articles in this journal are licensed under a Creative Commons Attribution 4.0 License. This journal is published by the University Library System, University of Pittsburgh and is cosponsored by the University of Pittsburgh Press. The Journal of Youth Development is the official peer-reviewed publication of the National Association of Extension 4-H Agents and the National AfterSchool Association. 
Accordingly, the authoring committee of the recent National Academies of Sciences, Engineering, and Medicine (NASEM) report, Promoting Positive Adolescent Health Behaviors and Outcomes: Thriving in the $21^{\text {st }}$ Century, ${ }^{1}$ used an optimal health framework to (a) identify the specific elements, or core components, of risk behavior prevention programs that successfully promote a variety of positive adolescent health outcomes, and (b) develop evidence-based recommendations for research and programs focused on adolescent health (NASEM, 2020).

The purpose of this article is to summarize the key messages and recommendations from that report that can be most useful to those working in youth-serving organizations. The first section provides key definitions and frameworks that guided the committee's work. The second section summarizes the results of the committee's systematic review, which was intended to identify the core components of programs and interventions that are effective across a variety of adolescent health behaviors and outcomes. The final section of this article presents a subset of the committee's recommendations and promising approaches that are useful to the diverse readership of this journal.

\section{Definitions}

\section{Optimal Health}

The statement of task for this report was developed with the study's sponsor, the Office of the Assistant Secretary for Health (OASH) of the United States Department of Health and Human Services (HHS), who requested that the committee review key questions related to the effective implementation of the teen pregnancy prevention (TPP) program through an optimal health lens. This charge is related to the OASH's mission to integrate the concept of optimal health into its projects and initiatives, particularly those related to sexual and reproductive health (U.S. Department of Health and Human Services, 2019).

\footnotetext{
${ }^{1}$ This article draws on previously published material from the National Academies of Sciences, Engineering, and Medicine Consensus Study report, Promoting Positive Adolescent Health Behaviors and Outcomes: Thriving in the $21^{\text {st }}$ Century, released in 2020 by the National Academies Press, Washington, D.C. Suggested citation for the full report: National Academies of Sciences, Engineering, and Medicine. 2020. Promoting Positive Adolescent Health Behaviors and Outcomes: Thriving in the $21^{\text {st }}$ Century. Washington, DC: The National Academies Press. https://doi.org/10.17226/25552. The report was authored by the Committee on Applying Lessons of Optimal Adolescent Health to Improve Behavioral Outcomes for Youth. Special thanks to Robert Graham, Angela Bryan, Tammy Chang, and Natacha Blain for reviewing the draft version of this manuscript. This study was funded by the Office of the Assistant Secretary of Health of the United States Department of Health and Human Services.
} 
In line with this first part of the statement of task, the committee needed to select a definition of "optimal health" to guide their work. However, the committee found only one definition in the peer-reviewed literature, which is the same definition used by the OASH for their optimal health model. This definition was first presented by O'Donnell in 1986 and later updated in his editorial statement for the American Journal of Health Promotion as "a dynamic balance of physical, emotional, social, spiritual, and intellectual health" (2009, p. iv).

Given the brevity of this definition in the original sources, and because it was not developed specifically for the adolescent health context, the committee looked to O'Donnell's more recent writing (2017) for further interpretation. Here, O'Donnell defines each of the five dimensions of his original definition (2017, p. 76):

physical health: the condition of the body

emotional health: the ability to cope with or avoid stress and other emotional challenges social health: the ability to form and maintain nurturing and productive relationships with family, friends, classmates, neighbors, and others

spiritual health: having a sense of purpose, love, hope, peace, and charity intellectual health: the necessary skills for academic achievements, career achievements, hobbies, and cultural pursuits

This more recent work also includes helpful interpretation. As described by O'Donnell, "It is not realistic to expect to reach that magic point of perfect balance and stay there. It is more realistic to seek opportunities for growth and think in terms of a process of striving for balance under changing circumstances" $(2017$, p. 76). The committee felt that this concept of seeking opportunities for growth under changing circumstances helped to make the important link to adolescent health and development, when exploration, risk taking, and experimentation play critical roles for brain and identity development. This definition was therefore used as the framework for their review of the adolescent health literature.

\section{Core Components}

The main focus of the statement of task was to identify what could be learned from other risk behavior programs that could be applied to all of the initiatives it oversees. To do this, the committee was charged with using a core components approach (Blase \& Fixsen, 2013). Briefly, the purpose of core components research is to identify the "active ingredients" of evidencebased programs (EBPs) or interventions rather than evaluating the program as a whole. As 
described in the report, core components can include intervention content, or the specific knowledge or actions that influence behavior (e.g., communication skills); processes, methods, or techniques that are used to deliver the content (e.g., modeling); locations and formats by which an intervention is delivered; and other implementation strategies that facilitate intervention delivery (e.g., provider training, availability of manuals; Barth \& Liggett-Creel, 2014; Chorpita et al., 2005; Embry \& Biglan, 2008; Hogue et al., 2017).

Once effective components are identified, they can be organized and combined in different ways to achieve the intended results. This more flexible structure can also lead to greater program fidelity compared to the original EBPs and help meet diverse community needs (Blase \& Fixsen, 2013). Furthermore, with regards to the committee's task, the identification of core components that are effective across health behaviors and programs could help coordinate programming efforts in areas that are traditionally siloed (e.g., teen pregnancy, substance use; U.S. Department of Health and Human Services, 2018).

The scientific literature suggests three main methods for identifying these core components. The first is the distillation and matching method, which uses algorithms to identify those techniques that can be used to individualize a treatment (Chorpita et al., 2005). The second is the Delphi technique, which convenes focus groups of experts who identify components through a consensus process (Garland et al., 2008). The third is meta-analysis and metaregression, which uses quantitative methods to analyze the relative effectiveness of program components in order to identify those that are most effective (Lipsey, 2018).

\section{The Committee's Systematic Review of Core Program Components}

\section{Strategy}

The committee's primary strategy for identifying these core components involved a systematic review of systematic reviews and meta-analyses. Although other methods were considered, including a meta-analysis of primary studies (Lipsey, 2018), the systematic review was chosen in order to examine the largest possible body of evidence in the available time.

The committee's methodological approach for extracting information from the articles was based on those used by core components researchers, as described above. More specifically, the committee used a consensus process to develop the model shown in Figure 1, which represents an organizing framework of the common features of adolescent health programs and 
interventions. ${ }^{2}$ Each article in the systematic review was coded based on these program features, and, if provided, effect sizes were used to assess the effectiveness of each of these components.

Figure 1. Organizing Framework Based on Committee Consensus Regarding the Features or Components of Programs or Interventions That Have the Potential to Promote Optimal Adolescent Health

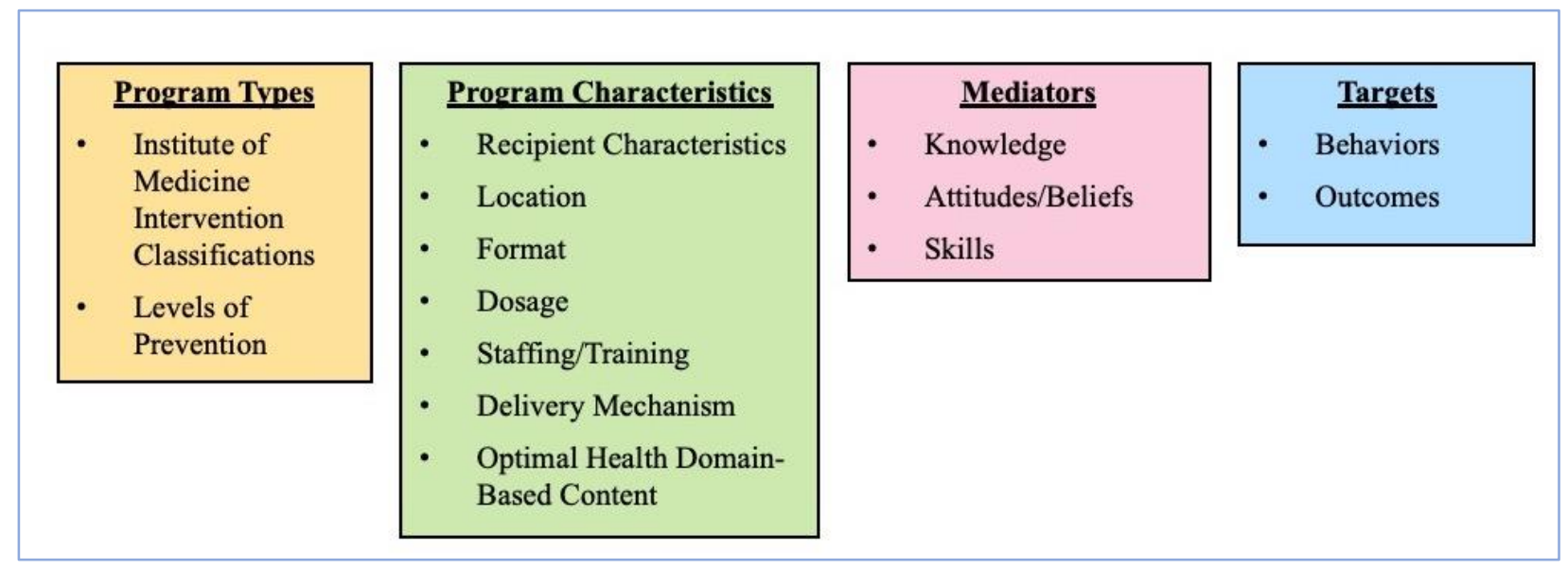

\section{Results}

From a database search that yielded 1,565 articles, the committee identified 31 that met its criteria for review. Unfortunately, the committee's ability to identify specific and discrete core program components was limited by the scope of the systematic reviews and meta-analyses that are currently available in the literature; many did not provide effect sizes as evidence of effectiveness for program components. However, several programs in these articles showed promising, common components across multiple domains of optimal health:

- Universal programs

- School-based

- Beginning in childhood

- Promoting social and emotional learning and positive youth development competencies

- Multiple sessions over longer periods of time

- Creating a supportive and inclusive culture in program settings

- Including diverse youth and their communities in program development, implementation, and evaluation efforts

\footnotetext{
${ }^{2}$ Further detail for each of these categories are available in Chapter 4 of the National Academies report.
} 
- Using theory-based approaches (e.g., social influence, social competence, cognitive behavioral therapy techniques)

In particular, the committee's review showed the important benefits of social-emotional learning and positive youth development programs, which provide children and adolescents with the building-block skills they need to make healthy decisions in a variety of situations throughout the life course.

\section{Recommendations and Promising Approaches}

The final charge to the committee was to provide recommendations for (a) a research agenda that incorporates a focus on optimal health for youth, and (b) improvements to OASH youthfocused programs. The OASH also requested ways that they could use their role to foster promising approaches in the initiatives it oversees, including mental and physical health, adolescent development, teen pregnancy, and reproductive health more broadly.

Although most of the committee's recommendations were directed towards federal government agencies, the key messages included in these recommendations and promising approaches are useful to other adolescent-serving audiences, including researchers, evaluators, program developers, and community organizations. All three of the committee's recommendations and their two promising approaches are detailed in the full report; those that are most relevant to positive youth development researchers and practitioners appear below in bold.

\section{The U.S. Department of Health and Human Services should fund additional research aimed at identifying, measuring, and evaluating the effectiveness of specific core components of programs and interventions focused on promoting positive health behaviors and outcomes among adolescents.}

As mentioned earlier, the identification of the core components of EBPs is a relatively new yet promising approach in the field of implementation science. However, most methods identify common components of effective programs without testing the effectiveness of each specific component. Although there have been several efforts to evaluate the core components of programs in practice settings (see Chorpita, et al. [2017; 2013] for children's mental health; Smith et al. [2012] for after-school programs; and Lipsey [2008], Lipsey et al. [2007], and Redpath \& Brander [2010]), these approaches have not yet been validated for adolescent 
health behaviors and outcomes more broadly. The committee therefore recommended that HHS fund research to further explore the use of core components approaches and to test whether those components do in fact result in better adolescent health outcomes compared to the original EBPs. If so, such components could be used to develop shorter and more focused interventions that would be (a) less costly and require less facilitator training, thus leading to greater program fidelity, and (b) more accessible to diverse populations than the original EBPs.

The Office of the Assistant Secretary for Health within the U.S. Department of Health and Human Services should fund universal, holistic, multi-component programs that meet all of the following criteria:

- promote and improve the health and well-being of the whole person, laying the foundation for specific, developmentally-appropriate behavioral skills development;

- begin in early childhood and are offered during critical developmental windows, from childhood throughout adolescence;

- consider adolescent decision making, exploration, and risk taking as normative;

- engage diverse communities, public policy makers, and societal leaders to improve modifiable social and environmental determinants of health and well-being that disadvantage and stress young people and their families; and - are theory-driven and evidence-based.

This recommendation was based on the results of the committee's systematic review. Using the optimal health framework showed how programs that are focused on social-emotional learning and positive youth development and begin in early childhood and continue through adolescence can be an effective way to lay the groundwork for promoting a variety of positive adolescent health behaviors. However, this recommendation for universal social-emotional learning and positive youth development programs does not mean that programs targeting specific health behaviors (e.g., substance abuse prevention, inclusive sex education) are not important. Rather, such social-emotional learning and positive youth development programs build a foundation of self-regulation, good decision-making, social awareness, and relationship skills upon which other programs that target specific risk behaviors, behavioral skills, and services (e.g., understanding social norms around drugs, negotiating condom use, reproductive health services) can be layered. 
Relatedly, this recommendation is informed by the research on adolescent risk-taking. In the report, the committee explained that adolescent risk taking is a normal part of the transition from parental or caregiver dependence to independence and self-identity. This is particularly relevant in the context of sexual development, whereby the necessary building blocks for adult relationships are established. As a part of their sexual development, adolescents must learn about themselves, their partners, and relationships in order to develop positive patterns for sexuality into adulthood (Harden, 2014; Tolman \& McClelland, 2011). As such, sexual activity during adolescence is not necessarily unhealthy or problematic. Rather, the key to healthy risk taking is to practice the foundational decision-making skills that allow adolescents to engage in less dangerous and more constructive risks. Therefore, instead of conceptualizing all risk taking as negative, programs can provide opportunities for adolescents to take healthy risks and make choices that will help them learn, grow, and thrive throughout their lives.

This recommendation also reflects the critical importance of reducing health disparities and promoting health equity by targeting the social determinants of health that disadvantage marginalized communities. Programs should continue to be developed and implemented with input and support from the communities they serve, as their insights will help identify the most pressing needs for their respective youth populations (see below for further detail).

The committee also recommended that these programs be theory based and informed by scientific research evidence. The committee's review showed that effective approaches were more likely to be theory based, address social influences and norms, incorporate cognitivebehavioral skills, and consist of multiple components. However, recognizing that much of the current scientific literature is not designed to evaluate the effectiveness of core program components, the committee also recommended that these programs continue to evolve based on future research.

\section{Programs can benefit from implementing and evaluating policies and practices that promote inclusiveness and equity so that all youth are able to thrive.}

As mentioned above, targeting the social determinants of health that disadvantage marginalized communities is critically important for reducing health disparities and promoting health equity in adolescent health programs. However, the committee asserted that all health education programs can benefit from implementing and evaluating policies and practices that promote cultural inclusiveness and equity. In particular, health programs need to address the structural 
inequities, including racism, sexism, classism, ableism, xenophobia, and homophobia, that lead to health inequities (NASEM, 2017; Williams \& Mohammed, 2013). Doing so will help to avoid systematically and counterproductively excluding youth who may benefit from these programs, thus leading to better overall outcomes.

\section{Programs can benefit from including youth of diverse ages, racial/ethnic backgrounds, socioeconomic status, rurality/urbanity, sexual orientations, sexes/genders, and disability/ability status in their decision-making processes.}

Partnering with youth to develop the policies and programs that impact their health and wellbeing is critical. Youth are experts in their own experiences and challenges, so understanding these experiences and challenges from their perspectives can help create policies and programs that effectively address their needs and remove barriers to their health promotion (Ford et al., 2012; Mirra \& Garcia, 2017; OECD, 2017; Powers \& Tiffany, 2006; Wyatt \& Oliver, 2016). Furthermore, civic engagement by youth can enhance the effectiveness of public policy by increasing their ownership and building consensus on key policies (Partridge et al., 2018); however, the success of these policies is undermined when researchers and policymakers do not authentically value their expertise (OECD, 2017). Thus, engaging diverse groups of youth as experts can draw on the reciprocal benefits for adolescents, researchers, and policymakers (Zeldin et al., 2013), and further supports our shared goal of promoting positive health behaviors and outcomes for all youth.

\section{References}

Barth, R. P., \& Liggett-Creel, K. (2014). Common components of parenting programs for children birth to eight years of age involved with child welfare services. Children and Youth Services Review, 40, 6-12. https://doi.org/10.1016/j.childyouth.2014.02.004

Blase, K., \& Fixsen, D. (2013). Core intervention components: Identifying and operationalizing what makes programs work. https://aspe.hhs.gov/report/core-intervention-components-identifyingand-operationalizing-what-makes-programs-work

Chorpita, B. F., Daleiden, E. L., Park, A. L., Ward, A. M., Levy, M. C., Cromley, T., Chiu, A. W., Letamendi, A. M., Tsai, K. H., \& Krull, J. L. (2017). Child STEPs in California: A cluster randomized effectiveness trial comparing modular treatment with community implemented treatment for youth with anxiety, depression, conduct problems, or traumatic stress. Journal of Consulting and Clinical Psychology, 85(1), 13-25. https://doi.org/10.1037/ccp0000133 
Chorpita, B. F., Daleiden, E. L., \& Weisz, J. R. (2005). Identifying and selecting the common elements of evidence based interventions: A distillation and matching model. Mental Health Services Research, 71 1), 5-20. https://link.springer.com/content/pdf/10.1007\%2Fs11020-005-1962-6.pdf

Chorpita, B. F., Weisz, J. R., Daleiden, E. L., Schoenwald, S. K., Palinkas, L. A., Miranda, J., HigaMcMillan, C. K., Nakamura, B. J., Austin, A. A., Borntrager, C. F., Ward, A., Wells, K. C., Gibbons, R. D., \& Research Network on Youth Mental Health. (2013). Long-term outcomes for the Child STEPs randomized effectiveness trial: A comparison of modular and standard treatment designs with usual care. Journal of Consulting and Clinical Psychology, 81(6), 999-1009. https://doi.org/10.1037/a0034200

Embry, D. D., \& Biglan, A. (2008). Evidence-based kernels: Fundamental units of behavioral influence. Clinical Child and Family Psychology Review, 11(3), 75-113. https://doi.org/10.1007/s10567-008$\underline{0036-x}$

Ford, T., Rasmus, S., \& Allen, J. (2012). Being useful: Achieving indigenous youth involvement in a community-based participatory research project in Alaska. International Journal of Circumpolar Health, 71(1), 18413. https://doi.org/10.3402/ijch.v71i0.18413

Garland, A. F., Hawley, K. M., Brookman-Frazee, L., \& Hurlburt, M. S. (2008). Identifying common elements of evidence-based psychosocial treatments for children's disruptive behavior problems. Journal of the American Academy of Child \& Adolescent Psychiatry, 47(5), 505-514. https://doi.org/10.1097/CHI.0b013e31816765c2

Harden, K. P. (2014). A sex-positive framework for research on adolescent sexuality. Perspectives on Psychological Science, 9(5), 455-469. https://doi.org/10.1177/1745691614535934

Hogue, A., Bobek, M., Dauber, S., Henderson, C. E., McLeod, B. D., \& Southam-Gerow, M. A. (2017). Distilling the core elements of family therapy for adolescent substance use: Conceptual and empirical solutions. Journal of Child \& Adolescent Substance Abuse, 26(6), 437-453. https://doi.org/10.1080/1067828X.2017.1322020

Lipsey, M. W. (2008). The Arizona Standardized Program Evaluation Protocol (SPEP) for assessing the effectiveness of programs for juvenile probationers: SPEP ratings and relative recidivism reduction for the initial SPEP sample. https://cdn.vanderbilt.edu/vu-my/wpcontent/uploads/sites/927/2013/04/14105643/AZ-Recidivism-Report final-revised.pdf

Lipsey, M. W. (2018). Effective use of the large body of research on the effectiveness of programs for juvenile offenders and the failure of the model programs approach. Criminology \& Public Policy, 171), 189-198. https://doi.org/10.1111/1745-9133.12345

Lipsey, M. W., Howell, J. C., \& Tidd, S. T. (2007). The Standardized Program Evaluation Protocol (SPEP): A practical approach to evaluating and improving juvenile justice programs in North Carolina. Final evaluation report. Vanderbilt University, Center for Evaluation Research and Methodology. 
Journal of Youth Development | http://jyd.pitt.edu/ | Vol. 15 Issue 3 DOI 10.5195/jyd.2020.949

Optimizing Adolescent Health Behavior Programs

Mirra, N., \& Garcia, A. (2017). Civic participation reimagined: Youth interrogation and innovation in the multimodal public sphere. Review of Research in Education, 41(1), 136-158. https://doi.org/10.3102/0091732x17690121

National Academies of Sciences, Engineering, and Medicine. (2017). Communities in action: Pathways to health equity. The National Academies Press. https://doi.org/10.17226/24624

National Academies of Sciences, Engineering, and Medicine. (2020). Promoting positive adolescent health behaviors and outcomes: Thriving in the $21^{\text {st }}$ century. The National Academies Press. https://doi.org/10.17226/25552

O'Donnell, M. P. (1986). Definition of health promotion. American Journal of Health Promotion, 1(1), 4-5. https://doi.org/10.4278/0890-1171-1.1.4

O'Donnell, M. P. (2009). Definition of health promotion 2.0: Embracing passion, enhancing motivation, recognizing dynamic balance, and creating opportunities. American Journal of Health Promotion, 24(1), iv. https://doi.org/10.4278/ajhp.24.1.iv

O'Donnell, M. P. (2017). Health Promotion in the workplace ( $5^{\text {th }}$ ed.). Art \& Science of Health Promotion Institute. https://www.artsciencehpi.com/books/health-promotion-workplace-5th-edition

OECD. (2017). Engaging youth in policy-making processes (Module 6). In Evidence-based policy making for youth well-being: A toolkit. OECD Publishing. https://doi.org/10.1787/9789264283923-10-en

Partridge, L., Astle, J., Grinsted, S., \& Strong, F. L. (2018). Teenagency: How young people can create a better world. https://www.thersa.org/discover/publications-and-articles/reports/teenagency-howyoung-people-can-create-a-better-world

Powers, J. L., \& Tiffany, J. S. (2006). Engaging youth in participatory research and evaluation. Journal of Public Health Management and Practice, 12, S79-S87.

https://journals.Iww.com/jphmp/Fulltext/2006/11001/Engaging Youth in Participatory Research and.15.aspx

Redpath, D. P., \& Brandner, J. K. (2010). The Arizona Standardized Program Evaluation Protocol (SPEP) for assessing the effectiveness of programs for juvenile probationers: SPEP rating and relative recidivism reduction; An update to the January 2008 Report by Dr. Mark Lipsey. https://cjjr.georgetown.edu/wp-content/uploads/2015/01/pres AZspep2010.pdf

Smith, C., Akiva, T., Sugar, S., Lo, Y.-J., Frank, K., Peck, S. C., Cortina, K. S., \& Devaney, T. (2012). Continuous quality improvement in afterschool settings: Impact findings from the Youth Program Quality Intervention study. http://cypq.org/sites/cypq.org/files/YPQITech\%20 2-29 12.pdf

Tolman, D. L., \& McClelland, S. I. (2011). Normative sexuality development in adolescence: A decade in review, 2000-2009. Journal of Research on Adolescence, 21(1), 242-255. https://doi.org/10.1111/j.1532-7795.2010.00726.x 
Journal of Youth Development | http://jyd.pitt.edu/ | Vol. 15 Issue 3 DOI 10.5195/jyd.2020.949 Optimizing Adolescent Health Behavior Programs

U.S. Department of Health and Human Services. (2018). Adolescent health: Think, act, grow $\mathbb{R}$ playbook. https://www.hhs.gov/ash/oah/sites/default/files/tag-playbook-2018.pdf

U.S. Department of Health and Human Services. (2019). Introducing the optimal health model. https://www.hhs.gov/opa/sites/default/files/Optimal-Health-Model-Overview.pdf

Williams, D. R., \& Mohammed, S. A. (2013). Racism and health I: Pathways and scientific evidence. American Behavioral Scientist, 578), 1152-1173. https://doi.org/10.1177/0002764213487340

Wyatt, Z., \& Oliver, L. (2016). Y-change: Young people as experts and collaborators. Advances in Social Work and Welfare Education, 18(1), 121-126.

Zeldin, S., Christens, B. D., \& Powers, J. L. (2013). The psychology and practice of youth-adult partnership: Bridging generations for youth development and community change. American Journal of Community Psychology, 51(3-4), 385-397. https://doi.org/10.1007/s10464-012-9558-y 